\title{
Study of the Improvement with Local Repair on AODV
}

\author{
${ }^{1}$ Mutian Zhu, *Ping Zong, ${ }^{2}$ Haotian Zhang, ${ }^{3}$ Dan Wu, ${ }^{4}$ Chengxiang Hua \\ ${ }_{1,}^{*}, 2,3,4$ College of Overseas Education, Nanjng University of Posts and Telecommunications, Nanjing \\ 210023, China \\ 1'mzhu03@foxmail.com, *zong@njupt.edu.cn, ²htpandog@foxmail.com, ${ }^{3}$ vvlll2flri@outlook.com, \\ 4hua_cx@163.com
}

Keywords: MANETs, AODV, Reactive Protocols, Local Repair, Chain Scission

Abstract. In this paper, we introduce a new improvement on AODV, which is a typical case of reactive protocols. One of the most important disadvantages of AODV is its long e2e delay, thus we focus more on the algorithm of reconnection when a chain scission happens. According the characteristic of AODV, each node knows that itself is the destination node or a immediate node. Hence we can leave out the process of broadcasting RREQ to inform the destination. So we can let the down-stream node broadcast a message that is similar to RREP to set up forward route directly to reduce the overhead and e2e delay. The result of simulation show that compared to original AODV, AODV using our improved method has less overhead and less e2e delay.

\section{Introduction}

Mobile Ad hoc Networks (MANETs) is composed of several mobile terminals. It is now becoming more and more popular in some situations where networks with fixed manufactures can not work any more such as military use and disasters recovery. In MANETs, nodes with high degree mobility can communicate with each other through wireless connections. Two nodes that are in the transmission range can communicate directly but the farther nodes need the help of immediate nodes to rebroadcast messages. The protocol in MANETs need to take the responsibility of discovering and renewing routes, and realize the communication between any two nodes with the help of immediate nodes. There have been many protocols proposed, but none of them have been accepted as standard yet.

Now there are mainly three types of protocols: proactive, reactive and hybrid protocols. In proactive protocols, nodes will exchange control messages with each other periodically to renew route tables. So the route table is always ready when it is needed and two nodes can communicate immediately. But a serious disadvantage is the giant overhead it consume to renew route tables. On the other hand, reactive protocols begin to set up routes only when there is a need. The disadvantage is that the delay before two nodes begin to communicate. There are also some hybrid protocols try to absorb both the advantages of proactive and reactive protocols.

The remainder of the passage is as follows: In section 2, first of all, we describe the original AODV protocol and explain how it works in a high degree mobility. Then we introduce some related works about AODV including the newest improvements on AODV. In section 3, based on the idea of "local repair" in section 2, we propose our new improvement to reduce overhead and e2e delay. Section 4 introduces the simulation result and section 5 gives a summary to our study.

\section{AODV}

\section{Construction of A Route}

Ad hoc On-demand Distance Vector Routing (AODV) is a kind of protocol that is designed for setting up or renewing routes in mobile Ad hoc networks (MANETs). It can realize both uni-cast and multicast. AODV is a typical case of reactive protocols.

AODV use two types of messages: RREQ (Route Request) and RREP(Route Reply) to discover and set up routes. A RREQ messages is used to discover routes. When a node receives a RREQ, it will 
store the information about the source node, the destination node and the node of the last hop. When the destination node, or a node that contains the route to the destination node receives a RREQ, it will choose a route to the source node with least hops and reply a RREP through it. Once the source node receive a RREP, a forward route se set up.

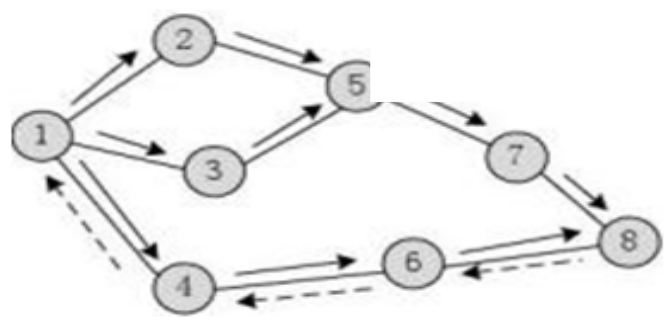

Fig 1 Process of discovering a route

Figure 1 shows the process of discovering and setting up a route. In the picture, node 1 is the source node and node 8 is the destination node. As the arrows show in the picture, when we need a route from node 1 to node 8 , first of all, node 1 will multicast RREQ to all of its neighbor nodes. Once a node receive a RREQ, it will store the information and then multicast it to its neighbor nodes as well. Until the destination node-- node 8 receive a RREQ, it will choose the route has the least hops (in this figure is 1 to 4 to 6 to 8 ) and reply a RREP, as the dotted line arrows show in the figure.Once node 1 receive the RREP, a forward route from node 1 to node 8 is set up. It can be seen from the process that in AODV, to set up a forward route, a network has to transmit messages back and forth on a route (RREQ and RREP). Because the node that receives a RREQ will store the information about the destination node and the source node, during the transmission, a node knows whether it is the destination node or a immediate node. This characteristic is significant and we will use it in the later improvement of AODV.

Because useless information can bring much burden to the network, a node will discard a message after a certain period. The period is called as Time To Live (TTL). When there is a break happened on a route, the source node will receive a RERR message and then reconstruct a route. Besides, each node will multicast a Hello messages to its neighbour periodically to get the latest information of its neighbour. Two methods can be used to maintain a route: ACK message (MAC layer) or HELLO message (Network layer).

\section{Repair}

Because the high mobility degree of nodes in MANETs, breaks on a route happen more frequently compare with the network with fixed manufactures. After a break happened, the network need to reconstruct a route according to the algorithm in the protocol and that is one of the most important resources of delay. In original AODV, the process of reconstruction is similar to construction: when a source node receive the RERR, it will multicast RREQ and start to find a route again. But the original method creates a huge delay. In the latest literature, a new method called "Local Repair" is introduced. The flow chart of the algorithm is shown as Figure 2. 


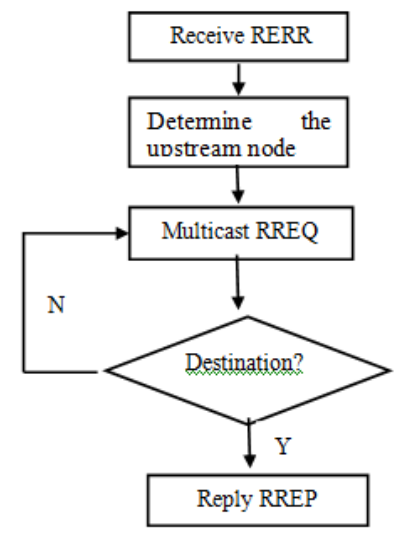

Fig 2 Local Repair

As shown in the chart, when a break happened, the upstream node before the broken part will multicast RREQ. This RREQ usually has a short TTL. When the destination node or a node that contains a route to the destination node, it will reply a RREP and construct a forward route. Compare to the original method, local repair method can reduce e2e delay in a steady environment and has higher efficiency. However, when the environment is not steady, or the topology structure is changing severely, the performance of local repair method is not quite satisfying anymore.

\section{The Method}

We mainly focus on the improvement of repairing the the break of a route. The main consequence brought by the high mobility degree nodes in the MANETs is more breaks. Hence compared with traditional network, MANETs need to consume more time and energy on maintaining the route table and rebuild routes. That is the main resource of delay and overhead. Consequently, to cut down e2e delay and overhead and raise the efficiency of MANETs, we mainly focus on the improvement on the algorithm of rebuilding a route when a break occurs.

The improvement is based on 2 main characteristics of AODV. First of all, in AODV, each node will store the information of the source node and the last hop node to generate a route table. That is to say, during the transmission, each node knows whether it is the source node or just a immediate node. Secondly, to build a forward route, AODV need to transmit messages twice on a route: RREQ forth and RREP back.

When a new route is to be built, the destination node doesn't know it is the destination. Hence AODV need RREQ to inform the destination node and let it response RREP to build forward route. That is the reason why AODV need to transmit messages twice when building a new route. We can see from the process that any time we need to build a forward route, RREP is always needed. However, when a break occurs during transmission, each node has had a route table, so the destination node has known that who it is. Thus we can skip the step of multicasting RREQ when rebuilding a route. We can let the downstream node at the break part multicast RREP to rebuild a forward route and cut down e2e delay and overhead. The new process is shown as Figure 3. 


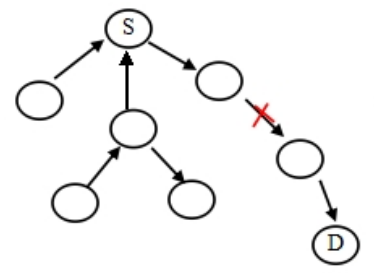

(a)

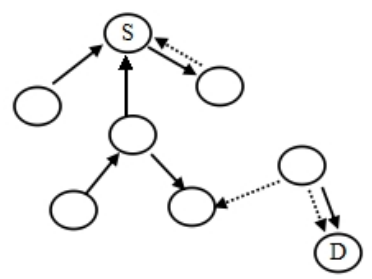

(b)

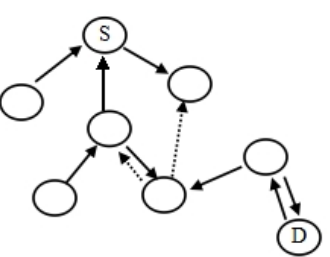

(c)

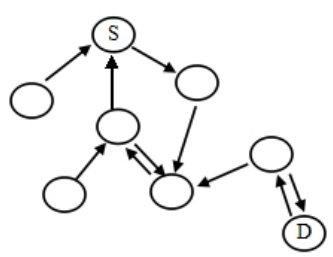

(d)

Fig 3 Modified Method

As can be seen from Fig 3, S is the source node and D is the destination node. In figure (b), solid arrows show the existing routes. When there is a break occurs, the upstream node at the break part will response a RERR to the source node. At the same time, the downstream node begins to multicast RERREP (Repair RREP) and the node that has received RERREP will continues to multicast it. As shown in figure (c), once the destination node or the up stream node (a node that contains a route to the source node) receives RERREP, a new forward route from $\mathrm{S}$ to $\mathrm{D}$ is built again. This new method skip the process of multicasting RREQ to reduce overhead and e2e delay.

\section{Simulation and Analysis}

\section{Simulation Environment}

We use ns-2 simulator to evaluate our method on networks. The simulation parameters are shown in Table 1.

Table 1 Simulation Parameters:

\begin{tabular}{ll}
\hline Parameter & Value \\
\hline Network Dimensions & $1000^{*} 1000$ \\
Number of nodes & 50 \\
Packet Traffic & CBR with 60 connections \\
Speed & $0-15 \mathrm{~m} / \mathrm{s}$ \\
Pause Time & $3 \mathrm{~s}$ \\
Transmission Range & $250 \mathrm{~s}$ \\
Simulation Time & $200 \mathrm{~s}$ \\
Mobility Model & Random waypoint \\
\hline
\end{tabular}

\section{Simulation Results}

In the simulation, we use the speed of nodes to describe the stability of the environment. Higher speed means that nodes in the network dimensions have a higher degree mobility and the topology structure is changing more fiercely. We simulated and compare the performance of local repair method and modified method in environment with different node speed. We evaluate e2e delay and overhead to figure out whether the modified method is more efficient.

Figure 4 describes the end-to-end delay of networks with varying node speed. As can be seen from the figure that when the node speed is less than $10 \mathrm{~m} / \mathrm{s}$, modified method has lesser end-to-end delay than local repair method. That means our method can, to some degree, reduce delay when the node speed is not quite fast. 


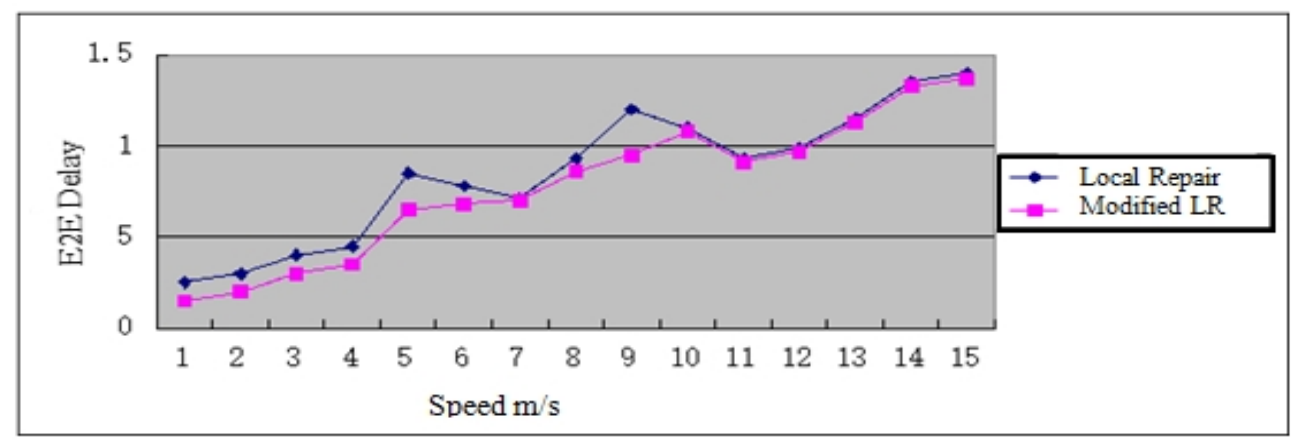

Fig 4 End-to-end Delay of Local Repair and Modified Method

Figure 5 demonstrates the overhead with different node speed. It can be seen from the chart that the overhead of modified method is always lesser than the original local repair method. That is to say, the modified method can face different situations and reduce overhead of the network.

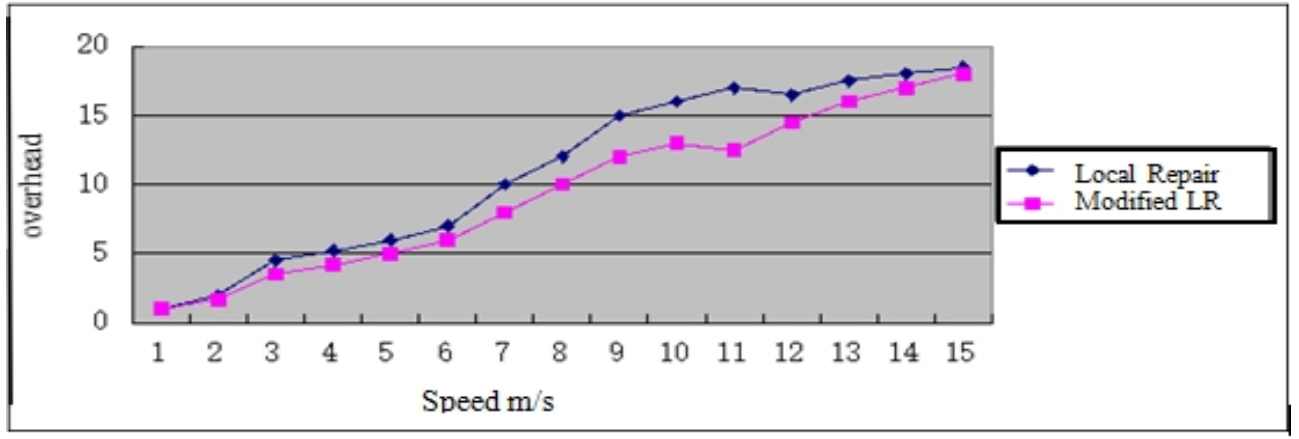

Fig5 End-to-end Delay of Local Repair and Modified Method

When facing different situations with different stability, the modified method successfully reduce the end-to-end delay and overhead. The network can work more efficiently when the environment is changing.

\section{Conclusion}

In this paper, we determine that compared with traditional network with manufactures, the main factor that affect the quality of MANETs is high probability of break during transmission. Hence we mainly focus on the algorithm of rebuilding a new route when a break occurs. In this paper, based on local repair, we introduce a new method without multicasting RREQ and rebuild a forward route directly in order to reduce end-to-end delay and overhead. According to the result of simulation, our new method can remarkably reduce end-to-end delay and overhead compared with local repair method. In the future, we will test other parameters of the modified AODV such as PDF and compared it with other protocols. Moreover, we would like to work out the best TTL of RERREP to make the network be more efficient. This research work is supported by the Science and Technology Innovation Training Program (STITP) of Jiangsu universities under grant No. SZDG2014039.

\section{References}

[1] Akkaya K, Younis M.A. Suvvery of rounting protocols in wireless sensoe networks[J]. Ad hoc Networks, 2005,3(3):325-349.

[2] Sunil Taneja, Ashwani Kush. A Survey of Routing Protocols in Mobile Ad Hoc Networks[J]. International Journal of Innovation, Management and Technology. 2010,1(3):279-283. 
[3] Wolfgang Kiess, Martin Mauve, A survey on real-world implementations of mobile ad-hoc networks. Ad hoc Networks 2007 (5):324-339.

[4] Loay Abusalah, Ashfaq Khokhar, Mohsen Guizani, A Survey of Secure Mobile Ad hoc Routing Protocols[J]. IEEE communications Surveys \& Tutorials. 2008,10(4): 78-93.

[5] Meilin Shi, Chun Ying. Protocols of [J]. Journal of Communication. 2001,22(11):93-103.

[6] Haitao Wang. Design of the structure of Ad hoc network[J]. China Data Communications. 2003(8):70-76.

[7] Luokun Liu, Yuan Zhang. Comparison of the Performance of AODV and DSDV[J]. Computer Simulation, 2006(2): 118-120.

[8] Haitao Wang. Ad hoc Network Technology and its Development Trend[J]. China Wireless Communications. 2004(9):18-21. 